\title{
Die Hausaufgabenvergabe im Unterricht: Eine Beobachtungsstudie an Grundschulen
}

\author{
Britta Kohler
}

Online publiziert: 27. Januar 2020

(C) Der/die Autor(en) 2020

Zusammenfassung Die Ausgestaltung der unterrichtlichen Hausaufgabenvergabe erscheint für das Gelingen der Hausaufgabenbearbeitung zentral. Sie wird vor allem wegen ihrer zeitlichen Platzierung im Unterrichtsverlauf und der damit einhergehenden Kürze und Hast seit Jahrzehnten kritisiert. Gleichzeitig stellt sie, insbesondere für die Grundschule, ein Forschungsdesiderat dar. Erstmals wurde deshalb die Hausaufgabenvergabe in 373 Unterrichtsstunden an 68 Grundschulen beobachtet und dokumentiert. Es zeigte sich einerseits eine Vielgestaltigkeit der Hausaufgabenvergabe und andererseits erwies sich die genannte Kritik als weiterhin berechtigt. Neben der im Unterrichtsverlauf späten Platzierung fiel insbesondere die geringe Zahl an differenzierten Aufgaben sowie Schülerfragen auf.

Schlüsselwörter Hausaufgabenvergabe · Unterrichtsqualität · Differenzierung · Zeit 


\title{
Assigning homework in the classroom: an observational study of elementary schools
}

\begin{abstract}
The way homework is given seems to be the key for its successful completion. It is especially the timing, brevity, and hurry of when and how homework is given in the classroom that is being criticized. At the same time, this problem of when and how to assign homework leads to a need for empirical research especially in the primary classes. That is why, for the first time, the way of giving homework was observed and documented in 373 class periods in 68 primary schools. Results show that on the one hand, there was a variety of ways homework was given and on the other hand, the abovementioned criticism was still found to be true. Problems noticed concern the assignment of homework towards the end of a class period and especially the small number of differentiated homework assignments as well as few student questions.
\end{abstract}

Keywords Homework assignment · Lesson quality · Differentiation · Time

\section{Einleitung}

Seit vielen Jahrzehnten finden sich in kritischen Auseinandersetzungen mit der Hausaufgabenthematik und auch in handlungsanleitenden Publikationen Hinweise darauf, dass die unterrichtliche Vergabe von Hausaufgaben häufig suboptimal ausgestaltet und in ihrer Relevanz unterschätzt wird. Kritisiert wird insbesondere die zeitliche Platzierung der Hausaufgabenvergabe ,im letzten Drittel der Unterrichtsstunde“ (Petersen et al. 1990, S. 29), welche häufig erst ,,vor oder nach Stundenende“ (Wollersheim 1985, S. 158) oder ,gerade mit dem Läuten“ (Unger 2009, S. 163) stattfinde (vgl. Grimm und Schulz-Gade 2015; Himmelrath 2015). In diesem Zusammenhang wird für Primar- und Sekundarstufe gleichermaßen formuliert, die Hausaufgaben würden oftmals „,noch ganz schnell“ (Geißler und Plock 1981, S. 59), ohne ,viel Zeit in Anspruch“ (Standop 2013, S. 149) zu nehmen, ,überhastet“ (Wagner 1956, S. 913) und ,gewissermaßen , aus dem Handgelenk geschüttelt ... ““ (Pakulla 1967, S. 47). Aus diesen Aussagen werden häufig Schlüsse im Hinblick auf die (mögliche) Qualität der unterrichtlichen Situation der Hausaufgabenvergabe gezogen: „In dieser Situation ist es kaum verwunderlich, wenn Schüler nur noch mit halbem Ohr zuhören, sich die Aufgaben nicht notieren und am Nachmittag nicht mehr wissen, was eigentlich zu bearbeiten war. Insbesondere nimmt sich der Lehrer bei dieser Praxis selbst die Möglichkeit, zusätzliche erklärende Erläuterungen zu geben oder Rückfragen der Schüler zu beantworten.“ (Petersen et al. 1990, S. 29; vgl. Unger 2009) Der Situation der Hausaufgabenvergabe wird aber nicht nur wenig Aufmerksamkeit im alltäglichen Unterricht zuteil, sondern sie stellt, wie weiter unten ausgeführt wird, auch ein Forschungsdesiderat dar. 


\section{Zur Relevanz und Qualität der unterrichtlichen Situation der Hausaufgabenvergabe}

Die Relevanz der Aufgabenstellung für die nachfolgende Aufgabenbearbeitung wird in der Allgemeinen Didaktik seit langem betont (z. B. Meyer 1997; vgl. Kleinknecht 2010). Für die Hausaufgabenbearbeitung erscheint die Situation der Aufgabenerteilung in besonderer Weise zentral, da Rückfragen während der Bearbeitung nicht mehr gestellt werden können und bei häuslicher Bearbeitung, anders als im Klassenzimmer, auch beiläufige Peer-Unterstützung nicht möglich ist. In der Hausaufgabenliteratur wird deshalb immer wieder gefordert, besonderes Augenmerk auf das Erteilen der Hausaufgaben zu legen (z. B. Hahn 1990; Kastens und Lipowksi 2010; vgl. Boßhammer und Schröder 2012).

Das Erteilen der Hausaufgaben im Unterricht ist von der Auswahl der Aufgaben analytisch zu trennen, auch wenn beides lokal und temporal nahezu zusammenfallen kann (siehe Abb. 1). Während es bei der Auswahl der Hausaufgaben um deren Funktion im Lernprozess geht und Kategorien wie Bedeutsamkeit, Komplexität oder kognitive Aktivierung (z.B. Kleinknecht 2010; Thonhauser 2019) und insbesondere fachdidaktische Fragen relevant sind, welche auch außerhalb des Unterrichts durchdacht werden können, stehen bei der Vergabe der Aufgaben Merkmale von Unterrichtsqualität im Vordergrund. Deutlich wird diese Unterscheidung auch beim „Prozessmodell zur Wirkungsweise von Hausaufgaben“ (Kohler 2017, S. 28 ff.), auf welches hier Bezug genommen werden soll. Es folgt einem engen Hausaufgaben-

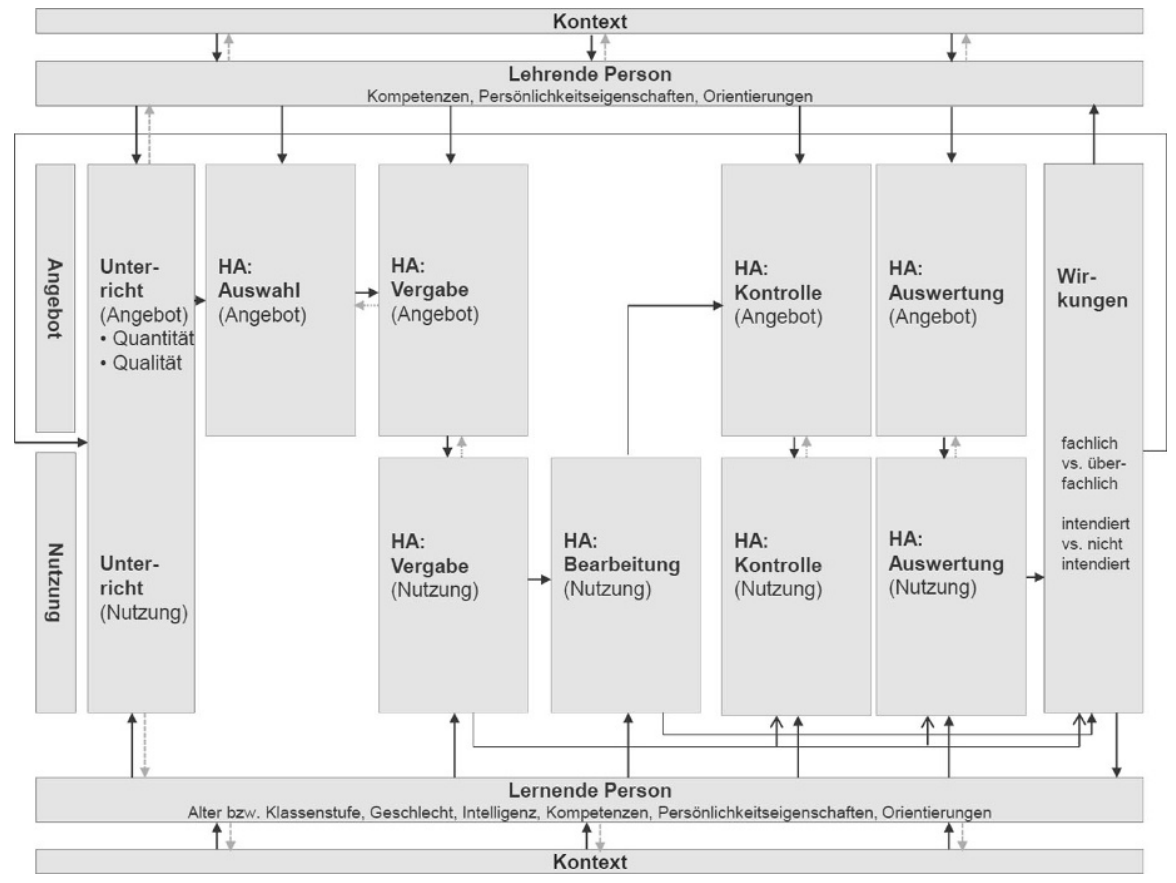

Abb. 1 Prozessmodell zur Wirkungsweise von Hausaufgaben (Kohler 2017, S. 28ff.) 
begriff und zeigt klar auf, dass die Vergabesituation eine Schlüsselsituation insofern ist, als sie am Anfang eines Prozesses steht, welcher, anders als bei Aufgabenbearbeitungen im Unterricht, keine Wiederholung der Vergabe bzw. keine spätere Anreicherung mit Hinweisen und abgestuften Hilfen bei misslingender Bearbeitung erlaubt.

Das allgemein als zentral anerkannte Unterrichtsqualitätsmerkmal der effektiven Zeitnutzung erfährt im Kontext der Hausaufgabenvergabe nun eine interessante Verschiebung: Während innerhalb der Unterrichtsqualitätsforschung (z. B. Helmke 2015) in der Regel ein Zeitmanagement favorisiert wird, bei dem es jede Minute $\mathrm{zu}$ nutzen gilt und einer Vergeudung von Lernzeit vorgebeugt werden soll, lautet hier die Aufforderung, sich bewusst mehr Zeit zu nehmen. Dennoch liegt auch diese Empfehlung nicht außerhalb der üblichen Argumentation, sondern kann mit Blick auf den gesamten Lernprozess als funktional im Sinne einer effektiven Zeitnutzung verstanden werden. So formulierte Aebli schon vor mehr als zwanzig Jahren mit Blick auf das Stellen komplexer Aufgaben- und Problemstellungen: „Es lohnt sich, dabei nicht mit der Zeit zu sparen. Der Ertrag wird den Zeit- und Energieaufwand mehrfach lohnen“ (Aebli 1998, S. 197).

\section{Forschungsbefunde zur Hausaufgabenvergabe}

Nach intensivierten Forschungsbemühungen in den letzten beiden Dekaden liegen zum Thema der Hausaufgaben inzwischen zahlreiche Forschungsbefunde aus dem nationalen und internationalen Raum vor. Bezieht man die verschiedenen Studien auf das vorgestellte Hausaufgabenmodell (Kohler 2017, S. $28 \mathrm{ff}$.), welches diese prozessbezogen zu ordnen vermag, so werden thematische Schwerpunkte der Hausaufgabenforschung genauso deutlich wie vorhandene Desiderate: Untersucht wurden in den letzten Jahren insbesondere die Aufgabenqualität als Teil der Aufgabenauswahl (z. B. Dettmers et al. 2010), die Hausaufgabenbearbeitung (z. B. Dettmers et al. 2011; Flunger et al. 2017; Haag und Mischo 2002; Perels et al. 2006; Wagner et al. 2010; Wild et al. 2006; Xu 2006), dabei auch die Frage der Quantität und Qualität sowie die Begründungen elterlicher Hilfe und Kontrolle und deren Relevanz (z. B. Dumont et al. 2014; Hoover-Dempsey et al. 2001; Pezdek et al. 2002; vgl. Tillack et al. 2015) und schließlich fachliche und überfachliche Effekte (z. B. Cooper et al. 2006; Hascher und Bischof 2000; Lipowsky et al. 2004; Roschelle et al. 2016; vgl. Haag und Streber 2015).

Kaum im Fokus waren dagegen bislang die unterrichtlichen Situationen der Vergabe sowie der Kontrolle und Auswertung von Hausaufgaben. Auch in Zusammenstellungen internationaler Forschung bzw. in Metaanalysen werden keine Befunde speziell zur Hausaufgabenvergabe ausgewiesen (z. B. Cooper et al. 2006; Fan et al. 2017; Hattie 2009). Aus dem deutschsprachigen Raum sind nur wenige, vorwiegend ältere und nicht durchweg nach heutigem Standard konzipierte und aufbereitete Befragungen und Beobachtungsstudien bekannt, deren zentrale Ergebnisse im Folgenden skizziert werden sollen. Sie gehen implizit und explizit davon aus, dass sich die von der späteren Unterrichtsqualitätsforschung benannten Merkmale von Unterrichtsqualität, wie z. B. Klarheit oder Motivierung, in der Situation der Hausaufga- 
benvergabe eher dann entfalten können, wenn ausreichend Zeit zur Verfügung steht. Letzteres entspricht auch neueren Konzepten und Befunden zur Unterrichtsqualität, welche aufzeigen, dass bestimmte, grundlegende Merkmale guten Unterrichts, wie z.B. Klassenführung, ,Voraussetzungen für die gelingende Umsetzung anderer Merkmale von Unterrichtsqualität sind“" (Pietsch 2013, S. 27; vgl. Pietsch 2010) und von daher bei der Entwicklung von Unterricht prioritär in den Blick genommen werden sollten.

Zwei ältere Fragebogenstudien weiten inhaltlichen Zuschnitts, die einerseits auf den Einschätzungen von ca. 300 niedersächsischen Lehrkräften verschiedener Schularten (Sommerla 1978) und andererseits auf den Angaben von 100 Hauptschullehrkräften aus Rheinland-Pfalz (Petersen et al. 1990) basieren, erbrachten, dass Hausaufgaben in der Regel im letzten Unterrichtsdrittel gestellt (vgl. Heinke und Salzwedel 1966) und hierfür überwiegend maximal fünf Minuten aufgewendet werden. Beide Untersuchungen fragten auch nach dem Zeitaufwand für das Stellen differenzierter Hausaufgaben und kamen gleichermaßen schlussfolgernd zu dem Ergebnis, diese seien wohl sehr selten bzw. seltener als angegeben, denn sie könnten ,nicht in 5 min sinnvoll entwickelt, erteilt und verstanden werden!“ (Sommerla 1978, S. 82; vgl. Petersen et al. 1990). Die Studie von Hascher und Hofmann (2008) mit 300 Lehrkräften der Sekundarstufe in Österreich und der Schweiz und auch jene von Standop (2013) mit knapp 150 Grundschullehrkräften aus NordrheinWestfalen konnten schließlich zeigen, dass es Lehrkräften sehr wichtig ist, Hausaufgaben so zu erklären, dass ihre Schülerinnen und Schüler sie tatsächlich verstehen. Allerdings zeigten sich Letztere in der Studie von Standop (a.a.O.) bezüglich der Frage, wie gut sie die Aufgabenstellungen verstünden, deutlich zurückhaltender als die Lehrkräfte. Diese auffallende Differenz zwischen den Angaben von Lehrenden und Lernenden konnte auch in einer eigenen Studie mit ca. 800 Schülerinnen und Schülern und etwa 40 Lehrkräften eines Gymnasiums repliziert werden: Sowohl das Item zur Aufmerksamkeit der Lernenden bei der Aufgabenerteilung als auch jenes zur Frage, ob die Hausaufgaben immer verstanden würden, erbrachte bei den Lernenden weitaus negativere Einschätzungen als bei den Lehrenden (Kohler 2017).

Wagner (1956) erhob keine Selbstauskünfte von Lehrkräften, sondern ließ die Hausaufgabenvergabe in der Sekundarstufe I in der ehemaligen DDR beobachten und dokumentieren. Aus insgesamt 106 Protokollen mit niedrig-inferenten Beobachtungen und wörtlich notierten Aussagen konnte er extrahieren, dass Hausaufgaben mehrheitlich kurz vor Stundenende und zu fast einem Drittel erst nach Stundenschluss gestellt wurden. Es zeigte sich, dass eine Hausaufgabenvergabe nach statt vor dem Klingelzeichen noch seltener Erläuterungen der Lehrkraft und Fragen der Schülerinnen und Schüler sowie ein Notieren der Aufgaben beinhaltete. Nur selten wurde von der Lehrkraft nach dem Verständnis gefragt, noch seltener wurde die Aufmerksamkeit der Lernenden explizit auf die Aufgabenvergabe gelenkt. Das Notieren der Hausaufgaben durch die Lernenden wurde in hohem Maße durch das Anschreiben seitens der Lehrenden determiniert; auch dieses fand bei einem Erteilen vor Stundenschluss häufiger als bei einem Erteilen nach Stundenschluss statt. Je jünger die Schüler waren, desto eher wurde auf das Notieren geachtet. Insgesamt fiel die Kritik des Autors an der Hausaufgabenvergabe der beobachteten Lehrkräfte deutlich negativ aus und er nahm mit seinen Befunden letztlich die kritischen Hinweise der 
didaktischen Literatur ab den 1960er Jahren vorweg. Eine spätere Pilotstudie mit dem Titel „Hausaufgaben als Indikator für die Qualität des Unterrichts“ (Neuper 1985) beinhaltete auf der Basis theoretischer Konzepte ähnliche Kategorien wie die Studie von Wagner (1956), allerdings ohne Bezugnahme auf diese und bei einer Stichprobe von vier Grundschullehrkräften auch mit anderer Intention.

Um nach mehr als 60 bzw. 30 Jahren aktuelle Daten von der zeitlichen Platzierung und Ausgestaltung der Hausaufgabenvergabe zu erhalten, wurde diese in einer eigenen Studie in 185 Unterrichtsstunden an 62 baden-württembergischen Gymnasien im Anschluss an Wagner (1956) und Neuper (1985) in den Jahren 2011 bis 2014 beobachtet und dokumentiert (Kohler 2015b). Es zeigte sich erwartungsgemäß, dass Hausaufgaben vorwiegend am und auch nach dem Stundenende gestellt wurden und die Vergabe bei etwa einem Viertel aller Fälle in die Pause hineinreichte. Die Situation nahm im Mittel knapp drei Minuten in Anspruch (in Doppelstunden knapp vier Minuten) und beinhaltete nur wenige Fragen seitens der Schülerinnen und Schüler. Je später das Erteilen der Hausaufgaben begann, desto kürzer fiel dieses aus. In höheren Klassen wurden weniger Fragen gestellt und die Aufgaben seltener notiert. Ob das Erteilen differenzierter Hausaufgaben mehr Zeit in Anspruch nimmt, konnte mangels differenzierter Hausaufgaben nicht überprüft werden, was Selbsteinschätzungen von Lehrpersonen zur Häufigkeit des Einsatzes dieser Aufgaben widerspricht (Kohler 2015a). Mit dieser Studie liegen nun aktuelle Beobachtungsdaten zur Hausaufgabenvergabe am Gymnasium vor. Diese stützen die Befunde von Wagner (1956) in hohem Maße. Unklar bleibt dabei die Frage der Übertragbarkeit der Ergebnisse auf die Grundschule, für die bislang keinerlei Beobachtungsdaten und auch kaum Befunde aus Befragungen vorliegen. Hinweise aus einer Befragungsstudie darauf, dass in der Primarstufe im Vergleich zur Sekundarstufe Hausaufgaben eher im ersten Stundendrittel und seltener nach Stundenende gestellt werden, sind vorzufinden, entstammen aber den 1960er Jahren (Heinke und Salzwedel 1966). Aktuell kann nur schwer prognostiziert werden, inwiefern die wenigen signifikanten Korrelationen mit der Klassenstufe in der Gymnasialstichprobe sich auch als Differenzen zwischen Primar- und Sekundarstufe abbilden und ob bzw. inwiefern strukturelle Unterschiede zwischen Grundschule und weiterführenden Schularten oder auch stufenspezifische Schul- und Unterrichtskulturen sich hier niederschlagen (vgl. Terhart 2011).

\section{Fragestellungen}

Ziel der Studie war es, die für die Bearbeitung von Hausaufgaben zentrale unterrichtliche Situation der Hausaufgabenvergabe erstmals an Grundschulen in den Blick zu nehmen und ihre Gestaltung in der alläglichen Praxis ökologisch valide mittels Beobachtung zu erfassen und mit ihren Merkmalen darzustellen. Entsprechend war die Qualität der einzelnen Lehrpersonenhandlungen nicht Gegenstand der Untersuchung. Die Auswahl der Beobachtungskategorien orientierte sich an den Forderungen der didaktischen Literatur sowie den oben dargestellten Befunden empirischer Untersuchungen und erfolgte parallel zu jener der eigenen Gymnasialstudie. Die Kategorien lassen sich zu vier Bereichen zusammenfassen, (1) dem Zeitpunkt, (2) dem Zeitbedarf, (3) den Lehrpersonenhandlungen bei der Hausaufgabenvergabe (Ange- 
botsseite; vgl. Abb. 1) und (4) den Verhaltensweisen der Schülerinnen und Schüler (Nutzungsseite; vgl. Abb. 1), und fanden bereits in der eigenen Gymnasialstudie Verwendung. Die einzelnen Fragestellungen lauteten wie folgt:

\subsection{Zeitpunkt der Vergabe innerhalb der Unterrichtsstunden}

- In welcher Unterrichtsminute beginnt und endet die Vergabe der Hausaufgaben?

Mit Bezug auf die didaktische Literatur (z. B. Grimm und Schulz-Gade 2015; Unger 2009) und vorhandene Beobachtungs- und Befragungsstudien der Sekundarstufe (Neuper 1985; Petersen et al. 1990; Wagner 1956) wird von einer Hausaufgabenvergabe vornehmlich am und auch nach dem Stundenende ausgegangen. Ob sich diese Befunde nahtlos auf die Primarstufe übertragen lassen, bleibt mit Blick auf die wenigen vorhandenen Befunde jedoch unklar (vgl. Heinke und Salzwedel 1966). Dabei kann vorab auch nicht eingeschätzt werden, wie eindeutig bestimmbar der Beginn der Hausaufgabenvergabe in der Grundschule ist und wie viele Unterrichtsstunden mit einem Klingelzeichen oder in anderer Weise klar abgegrenzt werden.

\subsection{Zeitbedarf für das Erteilen der Hausaufgaben}

- Wie viel Zeit wird für das Erteilen der Hausaufgaben benötigt?

- Hängen Zeitpunkt und Dauer der Vergabe miteinander zusammen?

- Gibt es einen Zusammenhang von Zeitbedarf und Klassenstufe?

- Gibt es einen Zusammenhang mit der Frage, ob die Aufgaben differenziert gestellt werden?

Ausgehend von Forderungen in der Hausaufgabenliteratur (z. B. Standop 2013; Unger 2009) und vorhandenen empirischen Befunden (Neuper 1985; Petersen et al. 1990; Sommerla 1978; Wagner 1956) wird erwartet, dass das Erteilen der Hausaufgaben durchschnittlich nur wenige Minuten in Anspruch nimmt und bei späterer Platzierung im Unterrichtsverlauf kürzer ausfällt (Kohler 2015b). Weiterhin wird vermutet, dass die Vergabe differenzierter Hausaufgaben mehr Zeit benötigt als die Vergabe undifferenzierter Aufgaben (Petersen et al. 1990; Sommerla 1978). Eine Unterteilung in verschiedene Formen der Differenzierung (z. B. Bohl et al. 2012) wird vorab nicht vorgenommen, da eine Klassifizierung im Prozess anspruchsvoll erscheint und überdies von einem eher geringen Anteil an differenzierten Aufgaben ausgegangen wird (vgl. z. B. Dettmers et al. 2009; Hascher und Hofmann 2008; Kohler 2015a, 2015b; Roßbach 1995; Standop 2013; Trautwein et al. 2006).

\subsection{Lehrpersonenhandlungen bei der Hausaufgabevergabe (Angebotsseite)}

- Welche Handlungen auf Seiten der Lehrkräfte werden bei den beobachteten Hausaufgabenvergaben wie häufig realisiert?

- Hängen die Häufigkeiten der Lehrpersonenhandlungen mit dem Zeitpunkt oder der Dauer der Hausaufgabenvergabe oder mit der Klassenstufe zusammen? 
Zunächst ist davon auszugehen, dass die Hausaufgaben angekündigt, benannt und zumeist auch erläutert werden und hierfür Aufmerksamkeit verlangt wird. Mit Blick auf die Untersuchung von Wagner (1956) und die eigene Gymnasialstudie (Kohler 2015b) wird weiterhin erwartet, dass Lehrkräfte eher selten zu Fragen auffordern. Eine (schwach) positive Korrelation der Zahl der erfassten Lehrpersonenhandlungen mit der Dauer der Hausaufgabenvergabe und ein (leicht) negativer Zusammenhang mit dem Zeitpunkt des Erteilens werden im Anschluss an Wagner (1956) und die eigene Studie (Kohler 2015b) vermutet. Bezüglich des Anschreibens und Notierens der Aufgaben und auch bezüglich der Zahl der Schülerfragen erscheint ein negativer Zusammenhang mit der Klassenstufe aufgrund der entsprechenden Befunde in der eigenen Sekundarstufenstudie möglich, mit Blick auf die geringere Altersstreuung in der Primarstufe einerseits und verschiedene Möglichkeiten adaptiven Lehrerverhaltens andererseits jedoch nicht zwingend.

\subsection{Verhaltensweisen der Schülerinnen und Schüler bei der Hausaufgabenvergabe (Nutzungsseite)}

- Stellen die Lernenden Fragen?

- Notieren die Schülerinnen und Schüler die Hausaufgaben?

- Sind die Lernenden bei der Hausaufgabenvergabe aufmerksam?

- Hängen die Verhaltensweisen der Schülerinnen und Schüler mit dem Zeitpunkt oder der Dauer der Hausaufgabenvergabe oder mit der Klassenstufe zusammen?

- Welche Zusammenhänge von Angebots- und Nutzungsseite zeigen sich bezüglich inhaltlich korrespondierender Handlungs- und Verhaltensweisen auf Seiten der Lehrenden und Lernenden?

Mit Bezug auf vorhandene empirische Befunde (Kohler 2015b; Wagner 1956) werden nur wenige Schülerfragen erwartet. Allerdings beziehen sich diese Befunde auf die Sekundarstufe und lassen somit spezifische Verhaltensweisen von Grundschulkindern außer Acht. Weiterhin wird davon ausgegangen, dass die Lernenden die Hausaufgaben überwiegend notieren und bei der Hausaufgabenvergabe (eher) aufmerksam sind. Vermutet wird, dass alle genannten Schülerverhaltensweisen mit dem Zeitpunkt (leicht negativ) und der Dauer (schwach positiv) der Hausaufgabenvergabe korrelieren. Inwiefern es hier Zusammenhänge mit der Klassenstufe gibt, kann nur schwer vorhergesagt werden, da durchaus gegenläufige Prozesse anzunehmen sind, wenn es beispielsweise um das Notieren von Hausaufgaben geht. Angebotsund Nutzungsseite sollten bezüglich inhaltlich korrespondierender Handlungs- und Verhaltensweisen auf der Seite der Lehrenden und Lernenden miteinander korrelieren, auch wenn dies in der Gymnasialstichprobe nur beim Notieren der Aufgaben der Fall war. 


\section{Methode}

\subsection{Stichprobe}

Die Studie basiert auf den Daten von 373 Hausaufgabenvergaben an 68 verschiedenen Grundschulen. Um diesen Stichprobenumfang realisieren zu können, hospitierten, beobachteten und dokumentierten 67 Lehramtsstudierende im Praktikum sowie Lehramtsanwärterinnen und -anwärter in mehr als 1500 Schulstunden, in denen mehrheitlich keine Hausaufgaben erteilt wurden. Die Beobachtungen der Hausaufgabenvergaben fanden in den Jahren 2017 und 2018 in Baden-Württemberg statt. Dokumentiert wurden im Wesentlichen Hausaufgabenvergaben in den Fächern Deutsch $(n=149)$ und Mathematik $(n=138)$, wobei die Aufgaben im Fach Deutsch zu $41 \%$ und im Fach Mathematik zu $62 \%$ dem Schulbuch entstammten. Die weiteren Hausaufgaben wurden, von Englisch (zehn Aufgaben) und Religion (drei Aufgaben) abgesehen, uneinheitlich klassifiziert und beispielsweise dem Fächerverbund „Mensch, Natur, Kultur“ (drei Aufgaben), aber auch dem Sachunterricht (30 Aufgaben) oder Anfangsunterricht (neun Aufgaben) zugesprochen. Etwa 15\% der Hausaufgaben wurden differenziert oder individuell gestellt (Deutsch: $n=22$; Mathematik: $n=17$; andere Fächer: $n=16$ ). Die Frage einer Differenzierung stand in keinem bedeutsamem Zusammenhang mit der Klassengröße $(r=-0,05, p=0,329)$, welche bei durchschnittlich 20,17 Schülerinnen und Schülern lag (SD=4,31). Offene Aufgaben, die eine Bearbeitung auf unterschiedlichen Niveaus und in unterschiedlichem Umfang gestatteten, wurden nicht als differenzierte Aufgaben klassifiziert (z. B. Textproduktion). Insgesamt zeigte sich eine große Vielfalt an Formen der Differenzierung und Individualisierung mit unterschiedlich großen Freiheitsgraden für die Lernenden.

Die Stichprobe verteilte sich weitgehend gleichmäßig über die Klassenstufen (Klasse 1: 17,2\%; Klasse 2: 22,9\%, Klasse 3: 18,3\%; Klasse 4: 22,9\%; ohne Angaben: $18,8 \%$ ). Pro Lehrkraft und pro Klasse wurde zumeist nur wenige Male erhoben; das Maximum pro Lehrkraft und Klasse lag bei 10 Erhebungen. Es handelt sich um eine Gelegenheitsstichprobe, welche vor allem aus der Bereitschaft der Lehrkräfte resultierte, Personen in studiengangbezogenen Blockpraktika und im Vorbereitungsdienst (,Referendariat“) regelmäßig im eigenen, weitgehend alltäglichen Unterricht hospitieren, beobachten und Daten erheben zu lassen. Die Beobachtung speziell der Hausaufgabenvergabe wurde mit Blick auf die angestrebte hohe ökologische Validität nicht angekündigt. Bei auftretenden Nachfragen konnten die Beobachtenden den Lehrkräften antworten, sie würden Aufgabenstellungen im Unterricht dokumentieren. Die weitaus meisten der besuchten Stunden hatten eine Länge von 45 Minuten; insgesamt 40 Stunden umfassten 60 bis 95 Minuten. In keinem Fall wurde eine Aufgliederung des Schultages in definierte Schulstunden unterlassen.

\subsection{Design und Ablauf}

Es beobachteten und dokumentierten insgesamt 67 Lehramtsanwärterinnen und -anwärter sowie Lehramtsstudierende höherer Semester, die sich mit den Beobachtungskategorien zuvor im Rahmen einer zweistündigen Schulung gründlich vertraut 
gemacht und die Erhebung auch mit Hilfe dreier beispielhafter und deutlich unterschiedlicher Videosequenzen ausführlich geübt hatten. Insbesondere waren unterschiedliche Beobachtungsergebnisse thematisiert und anhand der Videosequenzen diskutiert worden und hatten zu einem Papier mit Erläuterungen für Beobachtungen im Feld geführt. Auf zusätzliche Aufnahmegeräte (Audio oder Video) wurde verzichtet, da deren Präsenz das Verhalten der Lehrkraft hätte beeinflussen und somit die Daten hätte verzerren können.

Um eine möglichst hohe ökologische Validität zu erreichen, wurden die im Rahmen hochschulischer Blockpraktika oder innerhalb des Vorbereitungsdienstes regelmäßig stattfindenden Hospitationen dazu genutzt, die relevanten Merkmale der Hausaufgabenvergabe zu beobachten und zu notieren. Daraus resultierte leider, dass nur wenige Beobachtungen und Dokumentationen von zwei Personen gleichzeitig durchgeführt und damit Durchführungsobjektivität und Beurteilerübereinstimmung nur durch das vorab stattgefundene Training gesichert werden konnten. Entsprechend musste auch das Instrument hinreichend einfach und handhabbar gestaltet sein und sollte neben freien Feldern für Erläuterungen möglichst nur niedrig-inferente Kategorien umfassen (vgl. z. B. Kleinknecht 2010). In jenen Fällen, in denen zwei Personen die gleichen Hausaufgabenvergaben beobachteten, zeigten sich aber in der Regel nahezu vollständige Übereinstimmungen bei den Dokumentationen.

\subsection{Instrument}

Für die Beobachtung wurde ein nur einseitiger, klar strukturierter Beobachtungsbogen verwendet, welcher in fast identischer Weise bereits in der eigenen Gymnasialstudie erfolgreich eingesetzt worden war (Kohler 2015b). Er orientiert sich inhaltlich in hohem Maße an den Kategorien von Wagner (1956) bzw. den Indikatoren von Neuper (1985) und durfte ein gewisses Maß an Umfang und Komplexität nicht überschreiten, um eine möglichst objektive und vollständige Erfassung der zentralen Kategorien im Prozess zu gestatten. Diese Unterkomplexität schränkt die Differenziertheit der Erfassung und die Zahl möglicher Beobachtungskategorien ein, eignet sich aber durchaus zur Deskription der interessierenden Merkmale.

Erhoben werden sollten neben der Zeit des Beginns und des Endes der Vergabe auf der Angebotsseite acht dichotome Variablen (z. B. ,Lehrkraft kündigt Hausaufgabenvergabe an“) sowie die Zahl der beantworteten Schülerfragen. Auf der Nutzungsseite interessierten die Anzahl der Schülermeldungen, die Zahl der im Prozess gestellten Schülerfragen, der (geschätzte) Prozentsatz der Schülerinnen und Schüler, die die Hausaufgaben notierten, sowie die Aufmerksamkeit der Lernenden (fünfstufige Skala: $5=$ trifft zu; $1=$ trifft nicht $\mathrm{zu}$ ).

Mit Ausnahme des zuletzt genannten Items, dessen Ergebnisse nicht nur wegen der nötigen Interpretationsleistung, sondern auch aufgrund des fehlenden gemeinsamen Referenzrahmens nur mit Zurückhaltung interpretiert werden können, waren alle Beobachtungen niedrig-inferent angelegt. Dennoch kann nicht übersehen werden, dass auch scheinbar klare Kategorien wie „Lehrkraft kündigt Hausaufgabenstellung an (verbal oder nonverbal)“ eine gewisse Unschärfe aufweisen. Zudem erscheint das Erfassen beispielsweise der Zahl von Schülermeldungen und insbesondere der Häufigkeit der Notation im Prozess nicht ganz einfach. Die Studierenden 
und Referendare wurden deshalb, da eine Beobachtung im Tandem nur in wenigen Fällen möglich war, aufgefordert, bei Unklarheiten Notizen anzufertigen oder vorsichtshalber auf eine Kodierung zu verzichten. Außerdem wurden sie gebeten, die Hausaufgaben möglichst an den Beobachtungsbogen anzuheften oder die Aufgaben detailliert zu beschreiben. Personenbezogene Daten wurden nicht erhoben.

\section{Ergebnisse}

Die nachfolgend berichteten Ergebnisse beziehen sich auf die Gesamtstichprobe. Nur jene Ergebnisse, die den Zeitpunkt der Vergabe im Unterrichtsverlauf betreffen, werden ausschließlich für die 45-Minuten-Stunden berichtet.

\subsection{Zeitpunkt der Vergabe innerhalb der Unterrichtsstunden}

Der Beginn der Hausaufgabenvergabe konnte in allen Fällen bestimmt werden. Nahezu alle Lehrkräfte kündigten das Erteilen der Hausaufgaben explizit an und/oder nannten die Hausaufgabe (vgl. Tab. 1). Wie Abb. 2 zu entnehmen ist, begann die Vergabe der Hausaufgaben überwiegend im letzten Drittel der Unterrichtsstunde (Prozentsatz für die 45-Minuten-Stunden: 66,60\%). In etwa der Hälfte aller Stunden begannen die Lehrkräfte frühestens sechs Minuten vor dem Klingelzeichen (Prozentsatz für die 45-Minuten-Stunden: 51,20\%); etwa 5\% der Hausaufgabenvergaben fingen auch erst nach Stundenende an. Durchschnittlich begannen die Lehrkräfte in 45-Minuten-Stunden mit der Erteilung der Hausaufgaben in Minute 32,83 ( $\mathrm{SD}=13,22)$ und beendeten sie in Minute 37,22 ( $\mathrm{SD}=12,83)$. Die Hausaufgabenvergabe reichte bei ziemlich genau einem Viertel aller Fälle in die Pause hinein (Prozentsatz für die 45-Minuten-Stunden: 24,40\%).

\subsection{Zeitbedarf für das Erteilen der Hausaufgaben}

Die Hausaufgabenvergabe umfasste in 64,70\% aller Fälle zwei bis fünf Minuten und in $5 \%$ mehr als 10 Minuten $(\mathrm{MW}=5,32 \mathrm{~min} ; \mathrm{SD}=6,31)$. Je später Lehr-

Tab. 1 Prozentuale Häufigkeit erfasster Lehrpersonenhandlungen in der Situation der Hausaufgabenvergabe

\begin{tabular}{lccc}
\hline Lehrerhandlung & Trifft zu (\%) & Trifft nicht zu (\%) & $n$ \\
\hline L kündigt HA-Vergabe an & 92,40 & 7,60 & 368 \\
L verlangt Aufmerksamkeit & 81,50 & 18,50 & 367 \\
L nennt HA & 94,30 & 5,70 & 366 \\
L schreibt HA an & 69,50 & 30,50 & 367 \\
L erläutert HA & 66,00 & 34,00 & 368 \\
L fordert zu Fragen auf & 25,70 & 74,30 & 369 \\
L beantwortet Fragen & 39,10 & 60,90 & 362 \\
L fordert zum Notieren auf & 68,50 & 31,50 & 372 \\
L achtet auf Notation & 57,00 & 43,00 & 370 \\
\hline
\end{tabular}

Metrische Variable „L beantwortet Fragen“ dichotomisiert in „beantwortet vs. beantwortet nicht“ 


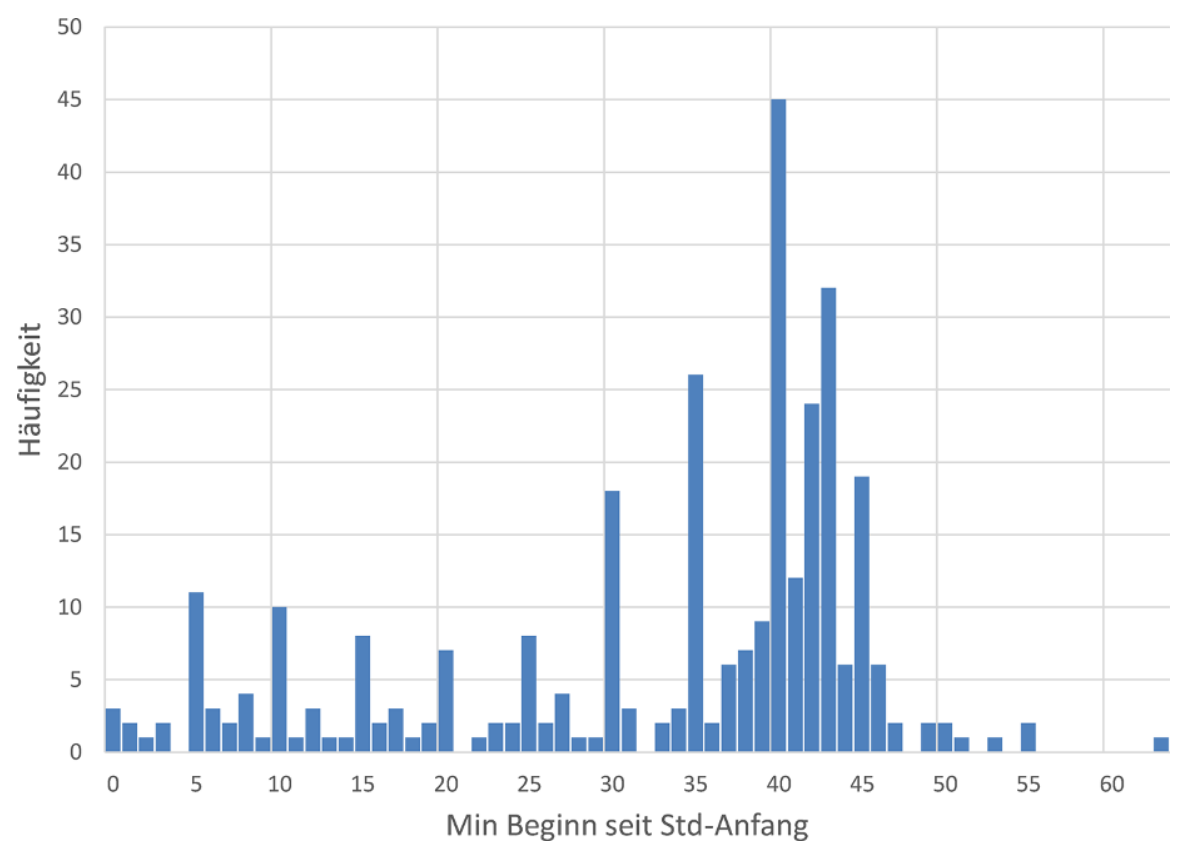

Abb. 2 Unterrichtsminute des Beginns der Hausaufgabenvergabe (45-Minuten-Stunden; $n=320$ )

kräfte mit dem Erteilen der Hausaufgaben begannen, desto kürzer fiel dieses aus $(r=-0,21, p<0,001)$. Ein statistisch bedeutsamer Zusammenhang mit der Klassenstufe $(r=-0,03, p=0,605)$ bestand nicht. Auch die Frage der Differenzierung blieb hier ohne Relevanz ( $r=0,01, p=0,847)$ : Während das Erteilen nicht differenzierter Aufgaben im Mittel 5,22 Minuten in Anspruch nahm, wendeten die Lehrkräfte bei differenzierten Hausaufgaben durchschnittlich 5,44 Minuten auf.

\subsection{Elemente der Situation der Hausaufgabevergabe (Angebotsseite)}

Tab. 1 können die Häufigkeiten entnommen werden, mit denen die erfassten Lehrpersonenhandlungen zu beobachten waren. Hierbei fällt auf, dass Handlungen, die den Beginn der Hausaufgabenvergabe und diese selbst markieren (ankündigen, Aufmerksamkeit verlangen, nennen) besonders häufig aufzufinden waren. Auffallend selten forderten die Lehrerinnen und Lehrer zu Rückfragen auf.

Zusammenhänge der verschiedenen Lehrpersonenhandlungen mit dem Zeitpunkt und der Dauer der Hausaufgabenvergabe sowie der Klassenstufe sind in Tab. 2 aufgeführt. Diese Zusammenhänge fallen insgesamt gering und weitgehend in der erwarteten Richtung aus. Es zeigt sich unter anderem, dass bei späterer Hausaufgabenvergabe weniger Schülerfragen beantwortet werden, während eine zeitlich umfangreichere Hausaufgabenvergabe unter anderem ein Mehr an Erläuterungen seitens der Lehrkraft bedeutet. Auch wird deutlich, dass in niedrigeren Klassenstufen die Hausaufgabenvergabe eher explizit angekündigt wird. 
Tab. 2 Korrelationen zwischen Elementen der Situation der Hausaufgabenvergabe, dem Zeitpunkt und der Dauer der Vergabe sowie der Klassenstufe

\begin{tabular}{|c|c|c|c|c|c|c|c|c|}
\hline & $\begin{array}{l}\text { Zeitpunkt } \\
\text { (Beginn) } \\
\text { der } \\
\text { Vergabe }\end{array}$ & $\begin{array}{l}\text { Dauer } \\
\text { der } \\
\text { Ver- } \\
\text { gabe }\end{array}$ & $\begin{array}{l}\text { Klas- } \\
\text { sen- } \\
\text { stufe }\end{array}$ & $\begin{array}{l}\text { L ver- } \\
\text { langt } \\
\text { Auf- } \\
\text { merk- } \\
\text { samkeit }\end{array}$ & $\begin{array}{l}\text { L } \\
\text { schreibt } \\
\text { HA } \\
\text { an }\end{array}$ & $\begin{array}{l}\text { L for- } \\
\text { dert zu } \\
\text { Fragen } \\
\text { auf }\end{array}$ & $\begin{array}{l}\text { L for- } \\
\text { dert } \\
\text { zum } \\
\text { Notieren } \\
\text { auf }\end{array}$ & $\begin{array}{l}\mathrm{L} \text { ach- } \\
\text { tet auf } \\
\text { Notati- } \\
\text { on }\end{array}$ \\
\hline $\begin{array}{l}\text { L kündigt } \\
\text { HA-Ver- } \\
\text { gabe an }\end{array}$ & $-0,098$ & $0,126^{*}$ & $-0,115^{*}$ & $0,188^{* *}$ & - & - & - & - \\
\hline $\begin{array}{l}\text { L nennt } \\
\text { HA }\end{array}$ & $0,265^{* *}$ & 0,001 & $-0,053$ & $0,131 *$ & - & - & - & - \\
\hline $\begin{array}{l}\text { L verlangt } \\
\text { Aufmerk- } \\
\text { samkeit }\end{array}$ & $-0,011$ & 0,009 & $-0,095$ & - & - & - & - & - \\
\hline $\begin{array}{l}\text { L schreibt } \\
\text { HA an }\end{array}$ & $-0,041$ & $-0,035$ & $0,175^{* *}$ & 0,075 & - & - & - & - \\
\hline $\begin{array}{l}\text { L erläutert } \\
\text { HA }\end{array}$ & $-0,011$ & $0,135^{*}$ & $-0,002$ & $0,149 * *$ & - & - & - & - \\
\hline $\begin{array}{l}\text { L fordert } \\
\text { zu Fragen } \\
\text { auf }\end{array}$ & 0,037 & $0,169^{* *}$ & $0,140 * *$ & $0,150^{* *}$ & - & - & - & - \\
\hline $\begin{array}{l}S \text { melden } \\
\text { sich }\end{array}$ & $-0,044$ & 0,041 & $0,114^{*}$ & $-0,017$ & - & $0,345^{* *}$ & - & - \\
\hline$S$ fragen & $-0,141^{*}$ & 0,092 & 0,086 & $-0,021$ & - & $0,173 * *$ & - & - \\
\hline $\begin{array}{l}\text { L beant- } \\
\text { wortet } \\
\text { Fragen }\end{array}$ & $-0,147^{*}$ & 0,099 & 0,085 & 0,025 & - & $0,201 * *$ & - & - \\
\hline $\begin{array}{l}\text { L fordert } \\
\text { zum } \\
\text { Notieren } \\
\text { auf }\end{array}$ & $-0,024$ & 0,028 & $-0,053$ & $0,143 *$ & $0,376 * *$ & - & - & - \\
\hline $\begin{array}{l}\text { L ach- } \\
\text { tet auf } \\
\text { Notation }\end{array}$ & $-0,064$ & 0,060 & $-0,002$ & $0,107 *$ & $0,357 * *$ & - & $0,625^{* *}$ & - \\
\hline $\begin{array}{l}\text { S notieren } \\
(\%)\end{array}$ & $-0,061$ & 0,006 & 0,084 & 0,099 & $0,577 * *$ & - & $0,801 * *$ & $0,681 * *$ \\
\hline $\begin{array}{l}\text { S sind } \\
\text { aufmerk- } \\
\text { sam }\end{array}$ & $-0,122^{*}$ & $-0,057$ & $-0,133^{*}$ & $0,289 * *$ & - & - & - & - \\
\hline
\end{tabular}

Signifikanzniveau: $*: p<0,05, * *: p<0,01$; zweiseitige Signifikanzprüfung

\subsection{Verhaltensweisen der Schülerinnen und Schüler in der Situation der Hausaufgabenvergabe (Nutzungsseite)}

In 30,6\% der Stunden meldete sich mindestens ein Schüler oder eine Schülerin in der Situation der Hausaufgabenvergabe ( $M W=0,63 ; \mathrm{SD}=1,21)$. Durchschnittlich wurden pro Klasse 0,84 Fragen gestellt ( $\mathrm{SD}=1,61$ ); das Maximum lag bei 15 Fragen. Im Mittel notierten 70,71\% (SD=39,28) der Schülerinnen und Schüler die gestellten 
Aufgaben. Ihre Aufmerksamkeit wurde auf einer fünfstufigen Skala ( $5=$ trifft zu; $1=$ trifft nicht $\mathrm{zu})$ als hoch $(\mathrm{MW}=4,17 ; \mathrm{SD}=0,96)$ eingeschätzt.

In Tab. 2 finden sich Zusammenhänge der genannten Variablen mit dem Zeitpunkt und der Dauer der Vergabesituation sowie mit der Klassenstufe. Die Zusammenhänge fallen durchweg gering und, sofern vorhanden, zumeist in der vermuteten Richtung aus. Signifikant negativ korrelieren unter anderem die Zahl der Fragen der Lernenden und ihre Aufmerksamkeit mit dem Zeitpunkt der Vergabe. Außerdem zeigt sich in höheren Klassenstufen eine geringfügig höhere Aufmerksamkeit.

Substantielle Zusammenhänge zeigen sich bei aufeinander bezogenen Lehrpersonenhandlungen (bezüglich Schülerfragen und Notation) sowie inhaltlich korrespondierenden Handlungen von Lehrenden und Lernenden: Sowohl bei der Höhe der Aufmerksamkeit als auch bei der Zahl der Meldungen und Fragen Lernender sowie bei der Häufigkeit der Notation der Aufgaben geht eine höhere Ausprägung auf Lernendenseite mit einer höheren Ausprägung auf Lehrendenseite einher.

\section{Diskussion}

Für die häusliche Bearbeitung von Hausaufgaben erscheint die unterrichtliche Situation der Hausaufgabenvergabe zentral. Während die didaktische Literatur dieser Situation seit vielen Jahrzehnten einen Mangel an Qualität zuschreibt, welcher insbesondere mit dem im Unterrichtsverlauf späten Zeitpunkt der Vergabe und der damit einhergehenden Kürze und Hast begründet wird, existieren, insbesondere für die Grundschule, hierzu kaum empirische Befunde. In einer Beobachtungsstudie an 68 baden-württembergischen Grundschulen wurden deshalb in den Jahren 2017 und 2018 insgesamt 373 Hausaufgabenvergaben in den Klassenstufen 1 bis 4 mittels Beobachtungsbogen in minimal invasiver und möglichst ökologisch valider Weise erfasst.

Es zeigte sich erwartungsgemäß, dass die Hausaufgaben vorwiegend am Ende der Stunde erteilt wurden, wobei etwa ein Viertel der Vergaben erst nach Stundenschluss beendet wurde. Damit wurden die aus der älteren Literatur und der eigenen Gymnasialstudie bekannten Befunde (Heinke und Salzwedel 1966; Kohler 2015b; Petersen et al. 1990; Sommerla 1978; Wagner 1956) im Wesentlichen repliziert, und es muss die in der Hausaufgabenliteratur seit Jahrzehnten formulierte Kritik an den ,,vor oder nach dem Stundenende“ (Wollersheim 1985) gestellten Hausaufgaben weiterhin aufrechterhalten werden. Für das Erteilen der Hausaufgaben wendeten die Lehrkräfte bei auffallend hoher Varianz durchschnittlich gut fünf Minuten auf, wobei die Klassenstufe ohne Bedeutung blieb. Erwartungsgemäß fiel die Vergabe bei einem späteren Beginn kürzer aus. Bei allen naheliegenden Hinweisen bezüglich der Gefahr einer überhasteten Vergabe am Stundenende sollte jedoch nicht übersehen werden, dass ein zügiges Erteilen bei hinreichend etablierten Routinen in der Klasse einerseits und gründlicher Erarbeitung der Aufgaben im vorausgehenden Unterricht andererseits oder auch bei sorgfältiger Vorbereitung im Rahmen der Unterrichtsvorbereitung seitens der Lehrkraft durchaus gelingen kann. So kann beispielsweise die Erläuterung eines Arbeitsblattes, dessen Struktur und Inhalt die vorangegangene Stunde abbildet, unter Umständen sehr zügig erfolgen. 
Ohne Relevanz für den zeitlichen Aufwand einer Hausaufgabenvergabe blieb die Frage der Differenzierung. Damit konnte die in der Hausaufgabenliteratur immer wieder formulierte Vermutung, für das Stellen differenzierter Hausaufgaben müsse im Unterricht zusätzlich Zeit investiert werden, nicht bestätigt werden. Nicht erfasst wurde allerdings die außerunterrichtliche Vorbereitungszeit für differenzierte vs. nicht differenzierte Aufgaben. Der in der Studie erhobene Anteil differenzierter Hausaufgaben erscheint mit $15 \%$ gering und widerspricht erwartungsgemäß vorhandenen Selbstauskünften zur Frage, wie häufig diese im eigenen Unterricht eingesetzt werden (z. B. Kohler 2015a; Roßbach 1995; Standop 2013; Trautwein et al. 2006; vgl. Heinzel und Koch 2017). Erkennbar wird damit nicht nur ein Auseinanderfallen von Selbstauskünften und extern erhobenen Daten, sondern auch die Tatsache, dass die bekannte Forderung der Hausaufgabenliteratur nach mehr Differenzierung bei den Hausaufgaben noch nicht abgegolten ist (z. B. Boßmann 1979; Geißler und Plock 1981). Gleichwohl zeigten sich in der Studie vielgestaltige Formen der Differenzierung und persönlichen Ansprache sowie der individuellen Zuweisung bzw. Auswahl von Hausaufgaben und auch ein höherer Anteil an differenzierten Hausaufgaben als an Gymnasien, an welchen in einer eigenen Studie differenzierte Hausaufgaben kaum aufzufinden waren (Kohler 2015b).

Zumeist wurde das Stellen der Hausaufgaben explizit angekündigt und als relevante Situation markiert. Dies gilt besonders für eine im Unterrichtsverlauf frühe Vergabe und mit Blick auf jüngere Schülerinnen und Schüler. In nur einem Viertel aller Fälle forderten die Lehrkräfte die Schülerinnen und Schüler zu Fragen auf und es wurden bei auffallend hoher Varianz pro Klasse im Schnitt nur 0,84 Fragen formuliert. Letzteres bestätigt, wie auch der nachfolgend genannte Befund, die Ergebnisse von Wagner (1956): Je später im Unterrichtsverlauf die Vergabe begann, desto weniger Fragen wurden von den Lernenden gestellt und von der Lehrkraft beantwortet. In mehr als zwei Drittel aller Hausaufgabenvergaben meldete sich niemand. Zeitlich umfangreichere Hausaufgabenvergaben zeichneten sich durch mehr Lehreraktivitäten, nicht aber durch mehr Schülerfragen aus. Beim Blick auf die Korrelationen zwischen den verschiedenen Variablen auf der Lehrerseite zeigte sich, dass diese nicht unabhängig voneinander variierten und beispielsweise Lehrkräfte, die Aufmerksamkeit verlangten, auch eher die Vergabe der Aufgaben explizit ankündigten. Klare Zusammenhänge von Angebots- und Nutzungsseite gab es beim Notieren der Hausaufgaben und auch bei der Aufforderung von Lehrkräften, Fragen zu stellen.

Abschließend kann resümiert werden, dass die vorliegende Studie einen ersten Einblick in die aktuelle und weitgehend alltägliche Praxis der Hausaufgabenvergabe an Grundschulen vor dem Hintergrund der vor mehreren Jahrzehnten erhobenen Beobachtungsdaten aus der Sekundarstufe I (Wagner 1956) sowie Ergebnissen einer neueren Gymnasialstudie (Kohler 2015b) bietet. Die Befunde verweisen einerseits auf eine vergleichsweise hohe Variabilität der Hausaufgabenvergabe in der Primarstufe, wie sie sich schon bei Heinke und Salzwedel (1966) gezeigt hatte, und andererseits auf eine hohe Konstanz dieser Vergabe über die Zeit und auch die Schularten hinweg. Es wird vielfach deutlich, dass die seit Jahrzehnten formulierte Kritik der Hausaufgabenliteratur am im Unterrichtsverlauf späten Erteilen von Hausaufgaben mit der möglichen Folge überhasteter Erläuterungen noch immer relevant ist. Folgt man der Idee eines gestuften Modells von Unterrichtsqualität (vgl. Pietsch 2010, 
2013), nach welchem „höherwertige Unterrichtsmerkmale“ (Pietsch 2013, S. 27) sich nur auf der Basis grundlegender Merkmale entfalten können, so erscheinen die hier dargestellten Befunde für die Lehrerbildung hoch bedeutsam.

Aus Forschungsperspektive bleiben aufgrund des Designs, welches vor allem auf eine hohe ökologische Validität zielte, dabei aber die Hausaufgabenvergabe nur bezüglich einiger Facetten erfassen konnte, noch viele Fragen offen. So kann auf der Basis der vorhandenen Daten beispielsweise nicht geklärt werden, wie die geringe Zahl an Schülerfragen zu deuten ist und warum zu allen Zeiten im Unterrichtsverlauf bei der Hausaufgabenvergabe so selten zu Fragen aufgefordert wurde. Ebenso bleibt offen, ob ein zeitlich wenig umfangreiches Erteilen von Hausaufgaben vielleicht sehr häufig mit hoher Struktur und Klarheit einhergeht und somit positiv einzuschätzen ist. Klären ließen sich offene Fragen insbesondere über vertiefte Analysen einzelner Hausaufgabenvergaben, beispielsweise mittels Videoanalysen, ihre Verknüpfung mit dem vorausgegangenen Unterricht sowie über qualitative Befragungen und auch über die anschließende Erfassung und Analyse der angefertigten Aufgaben sowie die Beobachtung der nachfolgenden Hausaufgabenbesprechung.

Danksagung Diese Forschungsarbeit wäre nicht möglich gewesen ohne die Unterstützung von Prof. Dr. Stefanie Schnebel (Pädagogische Hochschule Weingarten) sowie Sieglinde Opinc und Iris Meßner (Staatliches Seminar für Didaktik und Lehrerbildung Rottweil). Vielen Dank!

Funding Open Access funding provided by Projekt DEAL.

Open Access Dieser Artikel wird unter der Creative Commons Namensnennung 4.0 International Lizenz veröffentlicht, welche die Nutzung, Vervielfältigung, Bearbeitung, Verbreitung und Wiedergabe in jeglichem Medium und Format erlaubt, sofern Sie den/die ursprünglichen Autor(en) und die Quelle ordnungsgemäß nennen, einen Link zur Creative Commons Lizenz beifügen und angeben, ob Änderungen vorgenommen wurden.

Die in diesem Artikel enthaltenen Bilder und sonstiges Drittmaterial unterliegen ebenfalls der genannten Creative Commons Lizenz, sofern sich aus der Abbildungslegende nichts anderes ergibt. Sofern das betreffende Material nicht unter der genannten Creative Commons Lizenz steht und die betreffende Handlung nicht nach gesetzlichen Vorschriften erlaubt ist, ist für die oben aufgeführten Weiterverwendungen des Materials die Einwilligung des jeweiligen Rechteinhabers einzuholen.

Weitere Details zur Lizenz entnehmen Sie bitte der Lizenzinformation auf http://creativecommons.org/ licenses/by/4.0/deed.de.

\section{Literatur}

Aebli, H. (1998). Zwölf Grundformen des Lehrens (10. Aufl.). Stuttgart: Klett-Cotta.

Bohl, T., Kleinknecht, M., Batzel, A., \& Richey, P. (2012). Aufgabenkultur in der Schule. Baltmannsweiler: Schneider Hohengehren.

Boßhammer, H., \& Schröder, B. (2012). Von den Hausaufgaben zu Aufgaben in der Ganztagsschule. In S. Appel \& U. Rother (Hrsg.), Schulatmosphäre - Lernlandschaft - Lebenswelt (S. 67-81). Schwalbach, Taunus: Wochenschau.

Boßmann, D. (1979). Die verdammten Hausaufgaben. Frankfurt a.M.: Scriptor.

Cooper, H., Robinson, J.C., \& Patall, E. A. (2006). Does homework improve academic achievement? A synthesis of research, 1987-2003. Review of Educational Research, 76(1), 1-62.

Dettmers, S., Trautwein, U., \& Lüdtke, O. (2009). Eine Frage der Qualität? Die Rolle der Hausaufgabenqualität für Hausaufgabenverhalten und Leistung. Unterrichtswissenschaft, 37, 196-212. 
Dettmers, S., Trautwein, U., Lüdtke, O., Kunter, M., \& Baumert, J. (2010). Homework works if homework qualitiy is high: Using multilevel modeling to predict the development of achievement in mathematics. Journal of Educational Psychology, 102, 467-482.

Dettmers, S., Trautwein, U., Lüdtke, O., Goetz, T., Frenzel, A.C., \& Pekrun, R. (2011). Students' emotions during homework in mathematics: Testing a theoretical model of antecedents and achievement outcomes. Contemporary Educational Psychology, 36, 25-35.

Dumont, H., Trautwein, U., Nagy, G., \& Nagengast, B. (2014). Quality of parental homework involvement: Predictors and reciprocal relations with academic functioning in the reading domain. The Journal of Educational Psychology, 106(1), 144-161.

Fan, H., Xu, J., Cai, Z., He, J. M., \& Fan, X. (2017). Homework and students' achievement in math and science: a 30-year meta-analysis, 1986-2015. Educational Research Review, 20, 35-54.

Flunger, B., Trautwein, U., Nagengast, B., Lüdtke, O., Niggli, A., \& Schnyder, I. (2017). A person-centered approach to homework behavior: Students' characteristics predict their homework learning type. Contemporary Educational Psychology, 48, 1-15.

Geißler, E.E., \& Plock, H. (1981). Hausaufgaben - Hausarbeiten (3. Aufl.). Bad Heilbrunn: Klinkhardt.

Grimm, W., \& Schulz-Gade, G. (2015). Übungs- und Lernzeiten an der Ganztagsschule. Schwalbach, Taunus: Debus Pädagogik.

Haag, L., \& Mischo, C. (2002). Hausaufgabenverhalten: Bedingungen und Effekte. Empirische Pädagogik, 16(3), 311-327.

Haag, L., \& Streber, D. (2015). Hausaufgaben in der Grundschule. Zeitschrift für Grundschulforschung, $8(2), 86-99$.

Hahn, M. (1990). Serie: Grundkurs Unterrichtsgestaltung (14). Hausaufgabe in der Hauptschule. Lehrer Journal Hauptschulmagazin, 5(3), 60-62.

Hascher, T., \& Bischof, F. (2000). Integrierte und traditionelle Hausaufgaben in der Primarschule - ein Vergleich bezüglich Leistung, Belastung und Einstellungen zur Schule. Psychologie in Erziehung und Unterricht, 47, 252-265.

Hascher, T., \& Hofmann, F. (2008). Kompetenzbereich Hausaufgaben. In M. Gläser-Zikuda \& J. Seifried (Hrsg.), Lehrerexpertise (S. 143-164). Münster: Waxmann.

Hattie, J. (2009). Visible learning. London: Routledge.

Heinke, G., \& Salzwedel, W. (1966a). Intensivierung des Unterrichts und Hausaufgabenproblems. Pädagogik, 2, 2-11.

Heinke, G. \& Salzwedel, W. (1966b). Intensivierung des Unterrichts und Hausaufgabenproblems. Pädagogik, 2, 2-10.

Heinzel, F., \& Koch, K. (Hrsg.). (2017). Individualisierung im Grundschulunterricht. Anspruch, Realisierung und Risiken. Wiesbaden: Springer VS.

Helmke, A. (2015). Unterrichtsqualität und Lehrerprofessionalität (6. Aufl.). Seelze: Kallmeyer.

Himmelrath, A. (2015). Hausaufgaben - Nein danke! Bern: hep.

Hoover-Dempsey, K. V., Battiato, A.C., Walker, J. M. T., Reed, R.P., DeJong, J. M., \& Jones, K. P. (2001). Parental involvement in homework. Educational Psychologist, 36(3), 195-209.

Kastens, C., \& Lipowski, F. (2010). Hausaufgaben. In J. Mägdefrau (Hrsg.), Schulisches Lehren und Lernen (S. 109-126). Bad Heilbrunn: Klinkhardt.

Kleinknecht, M. (2010). Aufgabenkultur im Unterricht. Baltmannsweiler: Schneider Hohengehren.

Kohler, B. (2015a). „Das sollte ich eigentlich öfter tun“-Zur Praxis differenzierter Hausaufgaben aus der Sicht von Lehrkräften an Grundschulen und Gymnasien. Zeitschrift für Grundschulforschung, 8(2), $100-113$.

Kohler, B. (2015b). Die Vergabe von Hausaufgaben im Unterricht: Erste Daten zu einer vernachlässigten Schlüsselsituation. Empirische Pädagogik, 29(2), 189-210.

Kohler, B. (2017). Hausaufgaben. Überblick und Praxishilfen für Halbtags- und Ganztagsschulen. Weinheim: Beltz.

Lipowsky, F., Rakoczy, K., Klieme, E., Reusser, K., \& Pauli, C. (2004). Hausaufgabenpraxis im Mathematikunterricht. Ein Thema für die Unterrichtsqualitätsforschung? In J. Doll \& M. Prenzel (Hrsg.), Bildungsqualität von Schule (S. 250-266). Münster: Waxmann.

Meyer, H. (1997). Unterrichtsmethoden. II: Praxisband (8. Aufl.). Berlin: Cornelsen.

Neuper, W. (1985). Hausaufgaben als Indikator für die Qualität des Unterrichts. Unterrichtswissenschaft, $13(2), 140-148$.

Pakulla, R. (1967). Hausaufgaben. Berlin: VWV.

Perels, F., Löb, M., Schmitz, B., \& Haberstroh, J. (2006). Hausaufgabenverhalten aus der Perspektive der Selbstregulation. Zeitschrift für Entwicklungspsychologie und Pädagogische Psychologie, 38(4), 175-185. 
Petersen, J., Reinert, G.-B., \& Stephan, E. (1990). Betrifft: Hausaufgaben. Frankfurt a.M.: Peter Lang.

Pezdek, K., Berry, T., \& Renno, P. A. (2002). Children's mathematics achievement: the role of parents' perceptions and their involvement in homework. Journal of Educational Psychology, 94(4), 771-777.

Pietsch, M. (2010). Evaluation von Unterrichtsstandards. Zeitschrift für Erziehungswissenschaft, 13, $121-148$.

Pietsch, M. (2013). Was guten Unterricht kennzeichnet. bildung \& wissenschaft, 12, 24-27.

Roschelle, J., Feng, M., Murphy, R.F., \& Mason, C. A. (2016). Online mathematics homework increases student achievement. AERA open, 2(4), 1-12. https://doi.org/10.1177/2332858416673968.

Roßbach, H.-G. (1995). Hausaufgaben in der Grundschule. Ergebnisse einer empirischen Untersuchung. Die Deutsche Schule, 87(1), 103-111.

Sommerla, G. (1978). Praxis, Effektivität und Funktionen traditioneller Hausaufgaben im Urteil von Lehrern. In Niedersächsisches Kultusministerium (Hrsg.), Hausaufgaben - empirisch untersucht (S. 71-145).

Standop, J. (2013). Hausaufgaben in der Schule. Bad Heilbrunn: Klinkhardt.

Terhart, E. (2011). Grundschularbeit als Beruf. In W. Einsiedler, M. Götz, A. Hartinger, F. Heinzel, J. Kahlert \& U. Sandfuchs (Hrsg.), Handbuch Grundschulpädagogik und Grundschuldidaktik (3. Aufl. S. 128-138). Bad Heilbrunn: Klinkhardt.

Thonhauser, J. (2019). Das didaktische Potenzial von Lernaufgaben. In C. Schreiner, et al. (Hrsg.), Praxistransfer Schul- und Unterrichtsentwicklung (S. 153-170). Münster: Waxmann.

Tillack, C., Fischer, N., \& Kuhn, H.P. (2015). Hausaufgabenbetreuung in der Ganztagsschule. Zeitschrift für Grundschulforschung, 8(2), 114-130.

Trautwein, U., Lüdtke, O., Kastens, C., \& Köller, O. (2006). Effort on homework in grades 5-9: Development, motivational antecedents, and the association with effort on classwork. Child Development, 77(4), 1094-1111.

Unger, H.D. (2009). Hausaufgaben - ein notwendiger Bestandteil schulischen Lebens? In D. Bosse (Hrsg.), Gymnasiale Bildung zwischen Kompetenzerwerb und Kulturarbeit (S. 161-168). Wiesbaden: VS.

Wagner, K.-D. (1956). Zur Methodik der Erteilung von Hausaufgaben. Pädagogik, 11, 844-851.

Wagner, P., Hirtenlehner, H., Bacher, J., \& Schober, B. (2010). Zeitliche Belastung von Schülern durch die häusliche Arbeit für die Schule. Empirische Pädagogik, 24(1), 6-25.

Wild, E., Rammert, M., \& Siegmund, A. (2006). Die Förderung selbstbestimmter Formen der Lernmotivation in Elternhaus und Schule. In M. Prenzel \& L. Allolio-Näcke (Hrsg.), Untersuchungen zur Bildungsqualität von Schulen (S. 370-387). Münster: Waxmann.

Wollersheim, H.-W. (1985). Zur historischen Entwicklung und zum gegenwärtigen Stand der empirischen Hausaufgabenforschung. Die Realschule, 93(4), 158-165.

$\mathrm{Xu}$, J. (2006). Gender and homework management reported by high school students. Educational Psychology, 26, 73-91. 